Article

\title{
Tree Diversity and Tree Community Composition in Northern Part of Megacity Bengaluru, India
}

\author{
Baragur Neelappa Divakara ${ }^{1, *(\mathbb{D})}$, Chitradurga Umesh Nikitha ${ }^{1}$, Nils Nölke ${ }^{2}$, , Vindhya Prasad Tewari ${ }^{1} \mathbb{D}$ \\ and Christoph Kleinn ${ }^{2} \mathbb{B}$
}

check for

updates

Citation: Divakara, B.N.; Nikitha,

C.U.; Nölke, N.; Tewari, V.P.; Kleinn,

C. Tree Diversity and Tree

Community Composition in

Northern Part of Megacity Bengaluru,

India. Sustainability 2022, 14, 1295.

https://doi.org/10.3390/su14031295

Academic Editors: Eva Schlecht,

Uwe Altrock, Matthias Gassmann

and Andreas Thiel

Received: 17 December 2021

Accepted: 21 January 2022

Published: 24 January 2022

Publisher's Note: MDPI stays neutral with regard to jurisdictional claims in published maps and institutional affiliations.

Copyright: (c) 2022 by the authors. Licensee MDPI, Basel, Switzerland. This article is an open access article distributed under the terms and conditions of the Creative Commons Attribution (CC BY) license (https:// creativecommons.org/licenses/by/ $4.0 /)$.
1 Institute of Wood Science and Technology, Bengaluru 560003, Karnataka, India; thoshitha.cu@gmail.com (C.U.N.); tewarivp@gmail.com (V.P.T.)

2 Forest Inventory and Remote Sensing, Georg-August-University, 37073 Goettingen, Germany; nils.noelke@forst.uni-goettingen.de (N.N.); ckleinn@gwdg.de (C.K.)

* Correspondence: diwakarbn@icfre.org or bndsira@gmail.com
Abstract: Trees are natural capital assets, especially for cities, as they provide immense environmental benefits and improve urban biodiversity and ecology. However, urbanization has largely destroyed the original native ecosystems and has caused a homogenization where frequently native species are replaced by non-native species. When attempting to understand the role of trees in urban settings, it is important to generate science-based data on the spatial distribution of trees, their species composition and tree species diversity as a function of the degree of urbanization. Such information may specifically inform the planning of effective long-term management of trees across urban and rural gradients. A total of 23 of 1 ha each were surveyed along a Northern research transect laid out along the urban-rural gradient of the metropolitan area of Bengaluru, India. Plots were randomly selected from the stratum "settlement areas", where WorldView-3 imagery supported both stratification and plot selection. The plots were fully mapped for trees, where a total of eleven variables had been observed for each tree. In addition, the basal area and wood volume was calculated to understand the biomass potential of the trees in the plots. The diversity indices such as the Shannon index, Simpson index, Pielou's evenness and Margalef's richness were considered for comparing the species diversity, composition and distribution along the gradient of Bengaluru. A total of 1128 individuals of 93 tree species were recorded. Among 92 species identified along the northern gradient, 53 are exotic, and 39 are native species. The Shannon-Wiener index varied from 1.33 to 2.72; Simpson's index varied from 0.65 to 0.90; Pielou's index varied from 0.66 to 0.90 , and Margalef's index ranged from 1.41 to 5.20 along the gradient. The basal area increased from $96.39 \mathrm{~m}^{2}$ to $102.76 \mathrm{~m}^{2}$ from 2017 to 2019 along the transect, with a net gain of $6.37 \mathrm{~m}^{2}$. Similarly, the wood volume increased from $1819.57 \mathrm{~m}^{3}$ to $1926.23 \mathrm{~m}^{3}$ with a net gain of $106.66 \mathrm{~m}^{3}$. The present study reports on tree distribution, species composition and tree species diversity along a gradient from the city center to the rural surroundings of northern parts of Bengaluru city. The information generated may support the city planners/administrators by providing a holistic understanding of the species composition and abundance for a further selection of adaptive species and appropriate tree and vegetation management practices to conserve the existing green spaces and contribute towards sustainable urban planning. The sample plots laid out may also serve as permanent observation plots for monitoring the dynamics of tree cover in the city.

Keywords: species distribution; composition; diversity; urban-rural gradient; Bengaluru

\section{Introduction}

Urbanization is caused by population growth and population shifts from rural and surrounding areas to progressively developed towns or cities and is also a function of economic, political and geographical factors. Bengaluru is one among the cities, growing by $2.5 \%$ annually [1]. The city has experienced urbanization at a rapid pace, including through 
unplanned and uncontrolled developments, demographical expansion, heterogeneous land use, associated deforestation and other anthropogenic activities [2,3]. Currently, Bengaluru is the second-fastest growing and fifth-largest metropolis in India [2,4], with a current population of about 12.33 million in 2020 with an increase of $3.74 \%$ from the previous year [5].

The scientific evidence from the last two decades has emphasized the crucial necessity of green areas within urban ecological systems. However, urban planners and managers underestimate the role played by the trees [6,7]. Their role becomes even more important depending on the intensity of urbanization in the urban and peripheral regions of the sprawling city such as Bengaluru. The progressive developments in the city have negative impacts on biodiversity and ecosystem services, especially urban green spaces and green cover, particularly when trees and green spaces are not specifically and comprehensively considered during the planning of city development. The green spaces are then at high risk of experiencing loss, a decline in the area due to demand for urban expansion, and a lack of space for accommodating the existing and growing population $[8,9]$.

According to $[10,11]$, management and conservation of urban biodiversities may be supported by comprehensive data on urban trees, their distribution and species composition. Some studies on urban green spaces of Bengaluru have been carried out, focusing mainly on the urban areas, and they found that streets and parks are relatively low in density but high in species diversity when compared to other cities [6,12]. According to [13], the city had (approx. 705 parks) small, medium and also large-sized parks. Apart from parks and gardens, there were also 200 open spaces and green areas (roadside and avenue trees) that lacked sufficient infrastructure, and they can be considered for developing green spaces within the city limits [14]. According to [15] and [16], the estimated tree crown cover in the city area (at the respective points in time) was about $19.9 \%$, amounting to a per capita green space availability of about $17 \mathrm{~m}^{2}$.

A study by [12], with 127 sample plots, found that only $42 \%$ of the trees in the cities were native species. However, the parks of Bangalore are leading to homogenization, where every four out of five trees are exotic. This is in contrast to the parks in cities such as Potsdam (Germany) and Jeonju/Chonju (South Korea), where the native species are up to $81 \%$ and exotic species are less than $30 \%$ in the population, respectively $[17,18]$. The developmental activities such as road widening projects and encroachment have led to a significant loss in the proportion of prominent and mature large canopy trees, giving rise to urban heat islands [6]. The rapid expansion and growth of cities towards the urban periphery saw a phenomenal change in land use and land cover in Greater Bengaluru, which has resulted in a dramatic fragmentation of the landscape.

Thus, extending the study to the transition and the rural surroundings provides a holistic scenario of tree diversity and composition. Furthermore, it provides information on levels of urbanization in the rural-urban gradient, and data generated provide a better understanding of the species composition and abundance that can contribute towards sustainable urban planning and conservation for greater Bengaluru. Further, data generated may help appropriate tree and vegetation management practices through the selection of adapted species and compliance of safety standards along with proper planning and management for urban environments. Such information is beneficial for urban managers seeking to maximize the environmental benefits provided by trees and to analyze the critical impact of the environmental functions offered by these trees. In view of this, the present study focused on tree species diversity in the urban area along with the transition and the rural area of Bengaluru through spatial inventory.

\section{Methods}

\subsection{Study Area}

Bengaluru, the capital of State of Karnataka in India, is located in the south-eastern part of the Karnataka and geographically extends from $77^{\circ} 37^{\prime} 19.54^{\prime \prime}$ E and $12^{\circ} 59^{\prime} 09.76^{\prime \prime} \mathrm{N}$. Greater Bengaluru has an area of $741 \mathrm{~km}^{2}$ (2020). The city is subdivided into 8 zones with 
198 wards under the jurisdiction of Bruhat Bengaluru Mahanagara Palike (BBMP). The spatial extent of Bengaluru is experiencing substantial demographic expansion of its urban area over 10 times during the last five decades from $1949\left(69 \mathrm{~km}^{2}\right)$ to $2006\left(741 \mathrm{~km}^{2}\right)$ [4]. Population density of Bengaluru is 4378 persons per square kilometer.

\subsection{Data and Field Procedure}

Our study focused on trees in Northern research transects of Bengaluru City, defined and laid out by an Indian-German research consortium (Figure 1). Twenty-three plots of $100 \mathrm{~m} \times 100 \mathrm{~m}(1 \mathrm{ha})$ each were selected in the Northern transect. The selection of the field plots followed stratified random sampling: at first, the two strata "built-up" and "others" were distinguished where built-up was defined as those areas with more than $50 \%$ impervious surface, identified from WorldView-3 satellite images (Figure 2). We sampled only the stratum "built-up" as our main interest lies in urbanized areas.
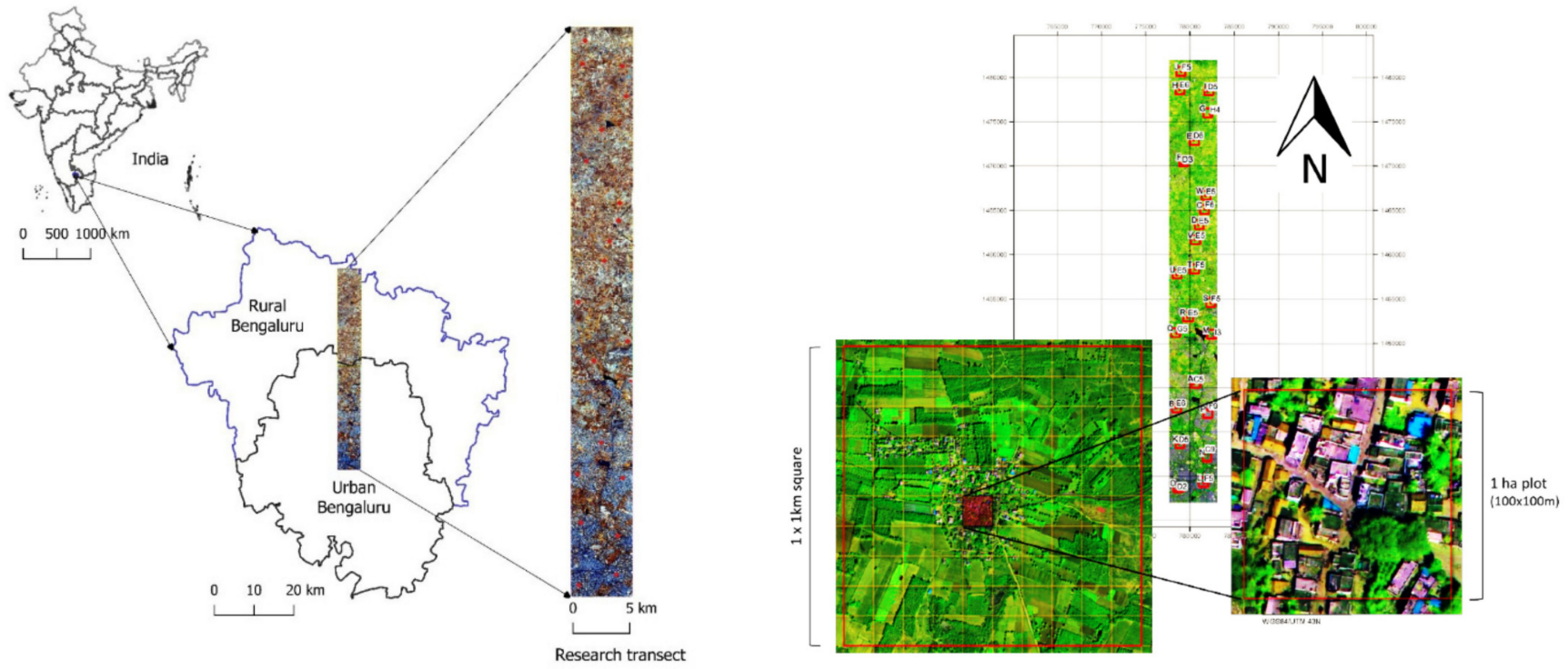

Figure 1. Location of the study area in the northern part of Bengaluru.
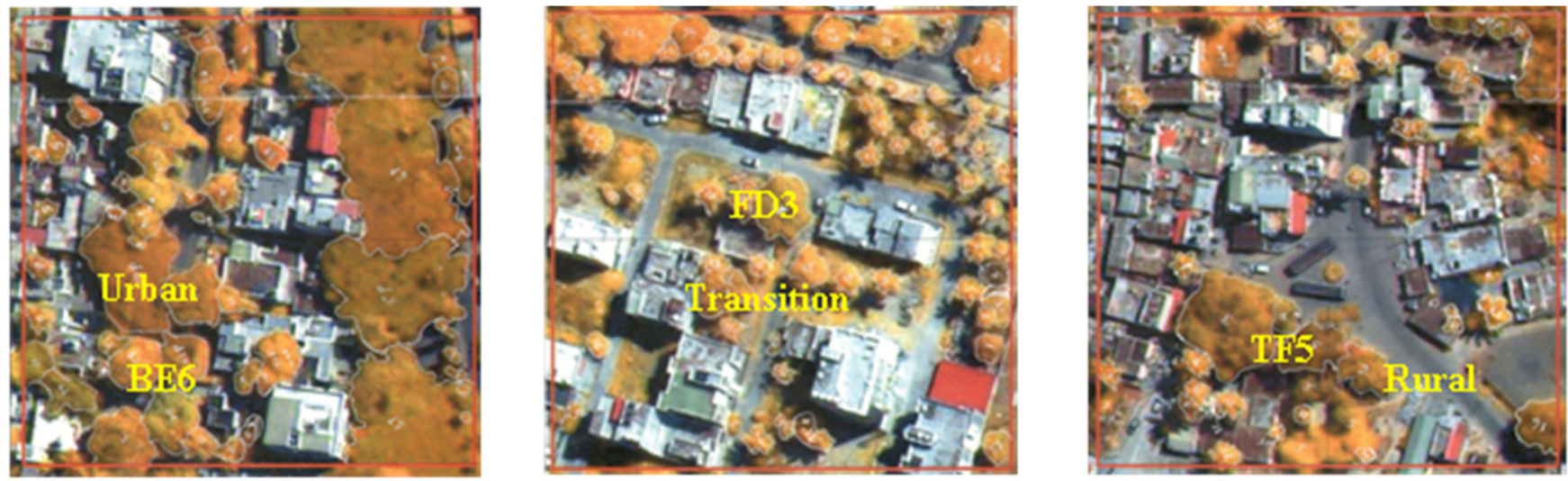

\section{Satellite images of the Northern transect}

Figure 2. WorldView-3 satellite image of 1 ha representing the three domains.

The 23 field plots were classified into the categories "urban", "transition", and "rural". This classification was performed by means of a pixel-wise analysis of the field plot from satellite imagery. The classification rules applied were: "urban" with $>50 \%$ built-up pixels, 
transition with $10-50 \%$ built-up pixels, and rural with $0-10 \%$ built-up pixels. The distance of each plot from the city center was also one of the criteria for the classification (Figure 2).

In the field plots, all trees $>10 \mathrm{~cm}$ dbh were tallied, including palms. The tree variables observed included, in addition to dbh, tree height, crown height and crown base height, are tree access, tree stand, tree permat, crown symmetry, crown shape, crown density and tree condition (on visual analysis). A detailed description of the tree variables is mentioned in Table 1. Further, using the measured dbh of the standing tree, basal area was calculated to observe the degree of stocking in the plots. In addition, the wood volume was also calculated to gain an idea about the above-ground biomass in the plots.

Table 1. Tree variables observed in the study.

\begin{tabular}{|c|c|c|c|}
\hline S1. No. & Tree Variables & Description & \\
\hline 1. & Diameter at Breast height DBH $(\mathrm{cm})$ & \multicolumn{2}{|c|}{ Tree stem was measured at $1.33 \mathrm{~m}$ above the ground with diameter tape. } \\
\hline 2. & Height (m) & \multicolumn{2}{|c|}{$\begin{array}{l}\text { Height is the total height of the standing tree measured as the straight line } \\
\text { distance from tip of the leading shoot to the ground level. }\end{array}$} \\
\hline 3. & Crown Base Height (m) & \multicolumn{2}{|c|}{ The average distance between ground and lowest foliage layer of the tree. } \\
\hline 4. & Crown Height (m) & \multicolumn{2}{|c|}{$\begin{array}{l}\text { It is the vertical measurement of the crown of a tree from the tip to the and } \\
\text { lowest foliage layer of the tree. }\end{array}$} \\
\hline 5. & Tree access & $\begin{array}{l}\text { It describes if the tree is directly } \\
\text { accessible or in fenced premises. }\end{array}$ & $\begin{array}{c}0=\text { accessible } \\
1=\text { not-accessible } \\
2=\text { tree stands on private property }\end{array}$ \\
\hline 6. & Tree stand & $\begin{array}{l}\text { It describes whether the tree is } \\
\text { solitary or part of a tree group. }\end{array}$ & $\begin{array}{l}0=\text { solitary tree } \\
1=\text { part of group of trees }\end{array}$ \\
\hline 7. & Tree permat & $\begin{array}{l}\text { It describes the pavement around the } \\
\text { tree stem. }\end{array}$ & $\begin{array}{c}1=\text { non-permeable } \\
2=\text { permeable pavements } \\
3=\text { bare soil }\end{array}$ \\
\hline 8. & Crown symmetry & $\begin{array}{l}\text { It describes the symmetry of } \\
\text { the crown. }\end{array}$ & $\begin{array}{c}0=\text { symmetrical } \\
1=\text { non-symmetrical }\end{array}$ \\
\hline 9. & Crown shape & $\begin{array}{l}\text { Based on visual estimate, crown } \\
\text { shape was noted based on } \\
\text { crown measurements. }\end{array}$ & $\begin{array}{c}1=\text { cylinder } \\
2=\text { horizontal ellipsoid } \\
3=\text { vertical ellipsoid } \\
4=\text { paraboloid } \\
5=\text { upside down paraboloid } \\
6=\text { sphere }\end{array}$ \\
\hline 10. & Crown density & $\begin{array}{l}\text { Based on visual assessment, the } \\
\text { crown hemisphere of the tree was } \\
\text { assigned with one of the } \\
\text { density classes. }\end{array}$ & $\begin{array}{c}0=\text { dense }(80-100 \%) \\
1=\text { medium }(40-80 \%) \\
2=\text { sparse }(0-40 \%)\end{array}$ \\
\hline 11. & Tree condition & $\begin{array}{l}\text { Based on a visual assessment, tree } \\
\text { health condition was noted down } \\
\text { based on four categories. }\end{array}$ & $\begin{array}{c}1=\text { healthy } \\
2=\text { affected } / \text { at risk } \\
3=\text { dying } / \text { declining } \\
4=\text { dead } \\
7=\text { Whole crown is visible } \\
8=\text { Crown only partially visible } \\
9=\text { Crown not visible }\end{array}$ \\
\hline
\end{tabular}

Vegetation composition was quantitatively evaluated for density, frequency and importance value index (IVI) according to [19]. The tree species diversity per sample plot was estimated from indices such as the Shannon-Wiener diversity index [20], Simpson's index of dominance [21], Margalef's Richness index [22] and Pielou's evenness index [23] were used (Table 2). The selected plots were revisited after one year to check the existence of marked trees and also to collect the dbh measurements of the tree species. The dbh measurements were taken to evaluate the growth of trees. Data for two consecutive years 
$(2018,2019)$ were collected to assess the temporal changes in the composition of marked trees for the northern transect.

Table 2. Indices of diversity used in the study.

\begin{tabular}{|c|c|c|c|c|}
\hline Sl. No. & Diversity Index & Formula & Range & Description \\
\hline 1 & $\begin{array}{l}\text { Shannon-Wiener diversity } \\
\text { index [20] }\end{array}$ & $H^{\prime}=-\sum_{i=1}^{S} P_{i}\left(\ln P_{i}\right)$ & $0-1$ & $\begin{array}{l}\text { It characterises both the number of } \\
\text { species and their distribution in } \\
\text { the community. } \\
\text { Higher value indicates high } \\
\text { diversity and vice versa. }\end{array}$ \\
\hline 2 & Simpson's index of diversity [21] & $D=1-\sum_{i=1}^{S}\left[n_{i} / N\right]^{2}$ & $0-1$ & $\begin{array}{l}\text { It give more emphasis on the most } \\
\text { abundant species in the community. } \\
\text { Higher value indicates high } \\
\text { diversity and vice versa. }\end{array}$ \\
\hline 3 & Simpson's index of dominance [21] & $C d=\sum_{i=1}^{S}\left[n_{i} / N\right]^{2}$ & & $\begin{array}{l}\text { It considers number of individual } \\
\text { species present, as well as the } \\
\text { relative abundance of each species. }\end{array}$ \\
\hline 4 & Margalef Richness index [22] & $R=\frac{S-1}{\ln N}$ & & $\begin{array}{l}\text { It is a measure of species richness in } \\
\text { the community. }\end{array}$ \\
\hline 5 & Pielou's evenness index [23] & $\mathrm{e}=\mathrm{H}^{\prime} / \ln \mathrm{S}$ & $0-1$ & $\begin{array}{c}\text { It is a measure of evenness in } \\
\text { the community. } \\
0 \text { stands for low and } 1 \text { for } \\
\text { high evenness. }\end{array}$ \\
\hline
\end{tabular}

Where, H'-Shannon's index of diversity, $P_{i}-n_{i} / N=$ Proportion of total sample belonging to the $i$ th species, $D$-Diversity, $n_{i}$-Number of individuals of the species ' $i$ ', $N$-Total number of individuals in the plot, $C d-$ Concentration of dominance, $R$-Margalef Richness index, $S$-Total number of species in a community, $n$-Total number of individuals observed.

The information on composition and diversity helps in better understanding both structural and functional dynamics of any ecosystem [24]. In specific, analyzing the diversity of species, vegetation composition, and the structure of any ecosystem assists in understanding ecological systems and also supports in developing sustainable management policies for improving and conserving the existing tree species in the ecosystem [25].

\section{Results}

In the present study, a total of 1128 individuals belonging to 93 species $(92$ species identified) were enumerated from 1 ha plots in the northern transect of Bengaluru. Tree species belonging to 39 families were recorded in the city (Table 3).

Table 3. Respective families of the tree species found along the gradients.

\begin{tabular}{ccc}
\hline S1. No. & Family & Northern Transect \\
\hline 1 & Anacardiaceae & 2 \\
2 & Annonaceae & 1 \\
3 & Apocynaceae & 2 \\
4 & Araucariaceae & 1 \\
5 & Arecaceae & 6 \\
6 & Bignoniaceae & 7 \\
7 & Boraginaceae & 2 \\
8 & Burseraceae & 1 \\
9 & Caricaceae & 1 \\
10 & Casuarinaceae & 1 \\
11 & Combretaceae & 1 \\
\hline
\end{tabular}


Table 3. Cont.

\begin{tabular}{|c|c|c|}
\hline Sl. No. & Family & Northern Transect \\
\hline 14 & Euphorbiaceae & 3 \\
\hline 15 & Fabaceae & 16 \\
\hline 16 & Lamiaceae & 1 \\
\hline 17 & Lauraceae & 1 \\
\hline 18 & Lecythidaceae & 1 \\
\hline 19 & Lythraceae & 2 \\
\hline 20 & Magnoliaceae & 1 \\
\hline 21 & Malvaceae & 2 \\
\hline 22 & Meliaceae & 5 \\
\hline 23 & Moraceae & 10 \\
\hline 24 & Moringaceae & 1 \\
\hline 25 & Muntingiaceae & 1 \\
\hline 26 & Myrtaceae & 5 \\
\hline 27 & Nyctaginaceae & 1 \\
\hline 28 & Oleaceae & \\
\hline 29 & Phyllanthaceae & 2 \\
\hline 30 & Podocarpaceae & 1 \\
\hline 31 & Proteaceae & 1 \\
\hline 32 & Rubiaceae & 2 \\
\hline 33 & Rutaceae & 5 \\
\hline 34 & Santalaceae & \\
\hline 35 & Sapindaceae & 1 \\
\hline 36 & Sapotaceae & 1 \\
\hline 37 & Strelitziaceae & 1 \\
\hline 38 & Unknown & 1 \\
\hline 39 & Verbenaceae & 1 \\
\hline \multicolumn{2}{|c|}{ Total } & 93 \\
\hline
\end{tabular}

Among 92 species identified along the northern gradient, 53 are exotic, and 39 were native species. The 23 plots of northern transect were categorized into eight plots of urban, four plots of transition and eleven plots of rural. The urban plots were comprised of 496 trees with 64 species, transition comprising of 180 trees with 37 species and rural plots comprising of 452 trees with 56 species.

Shannon-Wiener index varied from 1.33 to 2.72 with high and low observed diversity in plot 3 (NC9) and plot 21 (ID5), respectively. On the whole, the urban area had higher species diversity (2.42), followed by the transition (2.25) and rural area (1.99). Simpson's index varied from 0.65 to 0.90 , with high and low diversity observed in the urban plot (NC9) and rural (JF5), respectively, with an average value of 0.82 . Margalef's index also ranged from 1.41 to 5.20 , with an average index value of 3.65. The higher species richness was observed in the urban plot (KD5) and lower in the rural plot (ID5). Pielou's index varied from 0.66 to 0.90 with an average value of 0.81 (Table 4). The tree species were more or less evenly distributed along the urban-rural gradient. All the four diversity indices showed a decline towards transition and rural plots, indicating that the tree species were more diverse with species composition and distribution in urban plots. However, a sharp decline was observed towards transition and rural plots for the Shannon-Wiener and Margalef indexes. 
Table 4. Species diversity along the gradient of northern transect.

\begin{tabular}{|c|c|c|c|c|c|c|c|c|}
\hline Plot No. & Plot ID & $\begin{array}{l}\text { No. of } \\
\text { Trees }\end{array}$ & $\begin{array}{c}\text { No. of } \\
\text { Species }\end{array}$ & $\begin{array}{c}\text { Shannon- } \\
\text { Wiener } \\
\text { Diversity }\end{array}$ & $\begin{array}{l}\text { Simpson's } \\
\text { Dominance }\end{array}$ & $\begin{array}{c}\text { Simpson's } \\
\text { Diversity }\end{array}$ & Richness & Evenness \\
\hline 1 & OD2 & 42 & 10 & 1.893 & 0.206 & 0.794 & 2.408 & 0.822 \\
\hline 2 & LF5 & 49 & 20 & 2.577 & 0.111 & 0.889 & 4.882 & 0.860 \\
\hline 3 & NC9 & 103 & 25 & 2.720 & 0.092 & 0.908 & 5.178 & 0.845 \\
\hline 4 & KD5 & 47 & 21 & 2.656 & 0.108 & 0.892 & 5.195 & 0.872 \\
\hline 5 & PF9 & 48 & 16 & 2.285 & 0.157 & 0.843 & 3.875 & 0.824 \\
\hline 6 & BE6 & 89 & 18 & 2.325 & 0.151 & 0.849 & 3.787 & 0.804 \\
\hline 7 & AC5 & 61 & 18 & 2.358 & 0.163 & 0.837 & 4.135 & 0.816 \\
\hline 8 & MI3 & 57 & 21 & 2.592 & 0.106 & 0.894 & 4.947 & 0.851 \\
\hline 9 & QG5 & 37 & 14 & 2.229 & 0.154 & 0.846 & 3.323 & 0.869 \\
\hline 10 & RE5 & 63 & 17 & 2.468 & 0.114 & 0.886 & 3.862 & 0.871 \\
\hline 11 & SF5 & 35 & 12 & 2.024 & 0.187 & 0.813 & 3.094 & 0.814 \\
\hline 12 & UE5 & 45 & 17 & 2.283 & 0.160 & 0.840 & 4.203 & 0.806 \\
\hline 13 & TF5 & 48 & 10 & 1.658 & 0.272 & 0.728 & 2.325 & 0.720 \\
\hline 14 & VE5 & 36 & 11 & 1.830 & 0.225 & 0.775 & 2.791 & 0.763 \\
\hline 15 & DE5 & 49 & 12 & 1.947 & 0.207 & 0.793 & 2.826 & 0.784 \\
\hline 16 & CF6 & 26 & 12 & 2.086 & 0.189 & 0.811 & 3.376 & 0.839 \\
\hline 17 & WE5 & 44 & 17 & 2.444 & 0.123 & 0.877 & 4.228 & 0.863 \\
\hline 18 & FD3 & 76 & 19 & 2.287 & 0.172 & 0.828 & 4.156 & 0.777 \\
\hline 19 & ED6 & 19 & 12 & 2.233 & 0.147 & 0.853 & 3.736 & 0.899 \\
\hline 20 & GH4 & 52 & 15 & 2.395 & 0.115 & 0.885 & 3.543 & 0.884 \\
\hline 21 & ID5 & 17 & 5 & 1.335 & 0.315 & 0.685 & 1.412 & 0.829 \\
\hline 22 & HE6 & 50 & 14 & 1.979 & 0.230 & 0.770 & 3.323 & 0.750 \\
\hline 23 & JF5 & 35 & 13 & 1.685 & 0.349 & 0.651 & 3.375 & 0.657 \\
\hline
\end{tabular}

The species such as Cocos nucifera (100), Azadirachta indica (73.91), Mangifera indica (73.91), Artocarpus heterophyllus (65.22), Tectona grandis (56.52), Grevillea robusta (52.17) and Pongamia pinnata (52.17) were more frequently found along the gradient (Table 5). Tree species such as Cocos nucifera (11.91), Grevillea robusta (2.83), Pongamia pinnata (2.74), Polyalthia longifolia (2.48), Eucalyptus hybrid (2.13), Tectona grandis (2.00), Mangifera indica (1.65), Swietenia macrophylla (1.17), Ficus religiosa (1.04) and Tecoma stans (1.04) were densely populated along the gradient (Table 5). The Importance Value index (IVI) of each species in the northern transect is given in Table 6 .

Table 5. Species occurrence along the northern transect.

\begin{tabular}{|c|c|c|c|c|c|c|c|c|c|c|}
\hline $\begin{array}{l}\text { S1. } \\
\text { No. }\end{array}$ & List of Species & Origin & Urban & Transition & Rural & Total & Frequency & $\mathbf{R F}$ & Density & RD \\
\hline 1 & Acacia catechu & $\mathrm{Na}$ & 1 & 0 & 0 & 1 & 4.35 & 0.29 & 0.04 & 0.09 \\
\hline 2 & Acacia ferruginea & $\mathrm{Na}$ & 1 & 0 & 0 & 1 & 4.35 & 0.29 & 0.04 & 0.09 \\
\hline 3 & Aegle marmelos & $\mathrm{Na}$ & 0 & 0 & 1 & 1 & 4.35 & 0.29 & 0.04 & 0.09 \\
\hline 4 & Alangium salviifolium & $\mathrm{Na}$ & 0 & 0 & 1 & 1 & 4.35 & 0.29 & 0.04 & 0.09 \\
\hline 5 & Alastonia macrophylla & Ex & 3 & 0 & 0 & 3 & 4.35 & 0.29 & 0.13 & 0.27 \\
\hline 6 & Albizia kalkora & $\mathrm{Na}$ & 0 & 0 & 1 & 1 & 4.35 & 0.29 & 0.04 & 0.09 \\
\hline 7 & Anthocephalus kadamba & $\mathrm{Na}$ & 7 & 0 & 0 & 7 & 8.70 & 0.57 & 0.30 & 0.62 \\
\hline 8 & Araucaria cunninghamii & Ex & 5 & 0 & 2 & 7 & 21.74 & 1.43 & 0.30 & 0.62 \\
\hline 9 & Areca catechu & Ex & 3 & 4 & 2 & 9 & 21.74 & 1.43 & 0.39 & 0.80 \\
\hline 10 & Artocarpus heterophyllus & $\mathrm{Na}$ & 10 & 4 & 16 & 30 & 65.22 & 4.29 & 1.30 & 2.66 \\
\hline 11 & Azadirachta indica & $\mathrm{Na}$ & 7 & 10 & 15 & 32 & 73.91 & 4.86 & 1.39 & 2.84 \\
\hline 12 & Bauhinia purpurea & $\mathrm{Na}$ & 7 & 0 & 1 & 8 & 13.04 & 0.86 & 0.35 & 0.71 \\
\hline 13 & Bougainvillea glabra & Ex & 1 & 0 & 0 & 1 & 4.35 & 0.29 & 0.04 & 0.09 \\
\hline 14 & Callistemon lanceolatus & Ex & 1 & 0 & 0 & 1 & 4.35 & 0.29 & 0.04 & 0.09 \\
\hline 15 & Carica papaya & Ex & 1 & 1 & 7 & 9 & 13.04 & 0.86 & 0.39 & 0.80 \\
\hline 16 & Caryota urens & $\mathrm{Na}$ & 8 & 0 & 0 & 8 & 8.70 & 0.57 & 0.35 & 0.71 \\
\hline 17 & Cassia fistula & $\mathrm{Na}$ & 1 & 0 & 0 & 1 & 4.35 & 0.29 & 0.04 & 0.09 \\
\hline 18 & Casuarina equisetifolia & Ex & 2 & 0 & 0 & 2 & 4.35 & 0.29 & 0.09 & 0.18 \\
\hline
\end{tabular}


Table 5. Cont.

\begin{tabular}{|c|c|c|c|c|c|c|c|c|c|c|}
\hline $\begin{array}{l}\text { Sl. } \\
\text { No. }\end{array}$ & List of Species & Origin & Urban & Transition & Rural & Total & Frequency & RF & Density & RD \\
\hline 19 & Ceiba pentandra & $\mathrm{Na}$ & 0 & 0 & 5 & 5 & 8.70 & 0.57 & 0.22 & 0.44 \\
\hline 20 & Citrus limonum & $\mathrm{Na}$ & 0 & 0 & 1 & 1 & 4.35 & 0.29 & 0.04 & 0.09 \\
\hline 21 & Citrus maxima & Ex & 0 & 1 & 0 & 1 & 4.35 & 0.29 & 0.04 & 0.09 \\
\hline 22 & Citrus medica & $\mathrm{Na}$ & 0 & 0 & 1 & 1 & 4.35 & 0.29 & 0.04 & 0.09 \\
\hline 23 & Cocos nucifera & Ex & 84 & 46 & 144 & 274 & 100.00 & 6.57 & 11.91 & 24.29 \\
\hline 24 & Coffee arebica & Ex & 1 & 0 & 0 & 1 & 4.35 & 0.29 & 0.04 & 0.09 \\
\hline 25 & Commiphora caudata & $\mathrm{Na}$ & 0 & 0 & 5 & 5 & 13.04 & 0.86 & 0.22 & 0.44 \\
\hline 26 & Cordia dichotoma & $\mathrm{Na}$ & 0 & 3 & 3 & 6 & 17.39 & 1.14 & 0.26 & 0.53 \\
\hline 27 & Cordia mixa & $\mathrm{Na}$ & 1 & 0 & 0 & 1 & 4.35 & 0.29 & 0.04 & 0.09 \\
\hline 28 & Couroupita guianensis & Ex & 2 & 0 & 0 & 2 & 4.35 & 0.29 & 0.09 & 0.18 \\
\hline 29 & Crysalidocarpous lutescens & Ex & 0 & 0 & 1 & 1 & 4.35 & 0.29 & 0.04 & 0.09 \\
\hline 30 & Dead & Un & 1 & 1 & 0 & 2 & 8.70 & 0.57 & 0.09 & 0.18 \\
\hline 31 & Dilonix regia & Ex & 8 & 1 & 4 & 13 & 34.78 & 2.29 & 0.57 & 1.15 \\
\hline 32 & Duranta plumeri & Ex & 0 & 3 & 0 & 3 & 8.70 & 0.57 & 0.13 & 0.27 \\
\hline 33 & Eucalyptus hybrid & Ex & 5 & 9 & 35 & 49 & 43.48 & 2.86 & 2.13 & 4.34 \\
\hline 34 & Euphorbia synadenium & $\mathrm{Na}$ & 1 & 1 & 0 & 2 & 8.70 & 0.57 & 0.09 & 0.18 \\
\hline 35 & Euphorbia tirucalli & Ex & 0 & 0 & 1 & 1 & 4.35 & 0.29 & 0.04 & 0.09 \\
\hline 36 & Ficus benghalensis & $\mathrm{Na}$ & 0 & 1 & 3 & 4 & 8.70 & 0.57 & 0.17 & 0.35 \\
\hline 37 & Ficus benzamin & $\mathrm{Na}$ & 1 & 0 & 0 & 1 & 4.35 & 0.29 & 0.04 & 0.09 \\
\hline 38 & Ficus drupacea & $\mathrm{Na}$ & 0 & 1 & 6 & 7 & 13.04 & 0.86 & 0.30 & 0.62 \\
\hline 39 & Ficus elastica & $\mathrm{Na}$ & 2 & 0 & 0 & 2 & 4.35 & 0.29 & 0.09 & 0.18 \\
\hline 40 & Ficus glomerata & $\mathrm{Na}$ & 3 & 3 & 3 & 9 & 34.78 & 2.29 & 0.39 & 0.80 \\
\hline 41 & Ficus religiosa & $\mathrm{Na}$ & 1 & 6 & 17 & 24 & 43.48 & 2.86 & 1.04 & 2.13 \\
\hline 42 & Ficus tinctoria & $\mathrm{Na}$ & 0 & 0 & 4 & 4 & 4.35 & 0.29 & 0.17 & 0.35 \\
\hline 43 & Gliricidia sepium & Ex & 0 & 0 & 2 & 2 & 8.70 & 0.57 & 0.09 & 0.18 \\
\hline 44 & Grevillea robusta & Ex & 20 & 10 & 35 & 65 & 52.17 & 3.43 & 2.83 & 5.76 \\
\hline 45 & Jacaranda mimosifolia & Ex & 6 & 0 & 6 & 12 & 13.04 & 0.86 & 0.52 & 1.06 \\
\hline 46 & Kigelia pinnata & Ex & 1 & 0 & 0 & 1 & 4.35 & 0.29 & 0.04 & 0.09 \\
\hline 47 & Lagerstroemia flos-reginae & Ex & 4 & 1 & 0 & 5 & 13.04 & 0.86 & 0.22 & 0.44 \\
\hline 48 & Lannea coromandelica & $\mathrm{Na}$ & 0 & 0 & 1 & 1 & 4.35 & 0.29 & 0.04 & 0.09 \\
\hline 49 & Leucaena leucocephala & Ex & 0 & 2 & 0 & 2 & 8.70 & 0.57 & 0.09 & 0.18 \\
\hline 50 & Mangifera indica & $\mathrm{Na}$ & 23 & 6 & 9 & 38 & 73.91 & 4.86 & 1.65 & 3.37 \\
\hline 51 & Manilkara zapota & Ex & 1 & 0 & 0 & 1 & 4.35 & 0.29 & 0.04 & 0.09 \\
\hline 52 & Melia azedarach & $\mathrm{Na}$ & 1 & 0 & 1 & 2 & 8.70 & 0.57 & 0.09 & 0.18 \\
\hline 53 & Melia dubia & $\mathrm{Na}$ & 0 & 0 & 5 & 5 & 13.04 & 0.86 & 0.22 & 0.44 \\
\hline 54 & Michelia champaca & $\mathrm{Na}$ & 8 & 0 & 0 & 8 & 21.74 & 1.43 & 0.35 & 0.71 \\
\hline 55 & Millingtonia hortensis & Ex & 3 & 0 & 1 & 4 & 13.04 & 0.86 & 0.17 & 0.35 \\
\hline 56 & Moringa oleifera & $\mathrm{Na}$ & 5 & 4 & 6 & 15 & 30.43 & 2.00 & 0.65 & 1.33 \\
\hline 57 & Morus alba & Ex & 0 & 10 & 0 & 10 & 4.35 & 0.29 & 0.43 & 0.89 \\
\hline 58 & Morus papyrifera & Ex & 2 & 2 & 0 & 4 & 8.70 & 0.57 & 0.17 & 0.35 \\
\hline 59 & Muntingia calabura & Ex & 3 & 3 & 4 & 10 & 34.78 & 2.29 & 0.43 & 0.89 \\
\hline 60 & Murraya koenigii & $\mathrm{Na}$ & 1 & 4 & 13 & 18 & 39.13 & 2.57 & 0.78 & 1.60 \\
\hline 61 & Parkia biglandularis & Ex & 0 & 0 & 1 & 1 & 4.35 & 0.29 & 0.04 & 0.09 \\
\hline 62 & Peltophorum pterocarpum & $\mathrm{Na}$ & 21 & 0 & 1 & 22 & 21.74 & 1.43 & 0.96 & 1.95 \\
\hline 63 & Persea americana & Ex & 1 & 0 & 0 & 1 & 4.35 & 0.29 & 0.04 & 0.09 \\
\hline 64 & Phoenix dactylifera & Ex & 0 & 0 & 1 & 1 & 4.35 & 0.29 & 0.04 & 0.09 \\
\hline 65 & Phyllanthus acidus & Ex & 1 & 0 & 1 & 2 & 8.70 & 0.57 & 0.09 & 0.18 \\
\hline 66 & Phyllanthus emblica & $\mathrm{Na}$ & 1 & 0 & 1 & 2 & 8.70 & 0.57 & 0.09 & 0.18 \\
\hline 67 & Pithocellbium dulce & Ex & 1 & 0 & 0 & 1 & 4.35 & 0.29 & 0.04 & 0.09 \\
\hline 68 & Plumeria alba & Ex & 2 & 0 & 0 & 2 & 4.35 & 0.29 & 0.09 & 0.18 \\
\hline 69 & Podocarpus totara & Ex & 0 & 2 & 0 & 2 & 4.35 & 0.29 & 0.09 & 0.18 \\
\hline 70 & Polyalthia longifolia & Ex & 45 & 0 & 12 & 57 & 26.09 & 1.71 & 2.48 & 5.05 \\
\hline 71 & Pongamia pinnata & $\mathrm{Na}$ & 32 & 16 & 15 & 63 & 52.17 & 3.43 & 2.74 & 5.59 \\
\hline 72 & Prosopis juliflora & Ex & 0 & 1 & 0 & 1 & 4.35 & 0.29 & 0.04 & 0.09 \\
\hline 73 & Psidium guajava & Ex & 5 & 5 & 3 & 13 & 30.43 & 2.00 & 0.57 & 1.15 \\
\hline 74 & Punica granatum & Ex & 0 & 0 & 1 & 1 & 4.35 & 0.29 & 0.04 & 0.09 \\
\hline 75 & Ravenala madagascariensis & Ex & 2 & 0 & 0 & 2 & 4.35 & 0.29 & 0.09 & 0.18 \\
\hline 76 & Ricinus communis & Ex & 1 & 1 & 1 & 3 & 13.04 & 0.86 & 0.13 & 0.27 \\
\hline 77 & Royestonea regia & Ex & 2 & 0 & 1 & 3 & 8.70 & 0.57 & 0.13 & 0.27 \\
\hline 78 & Samanea saman & Ex & 11 & 1 & 4 & 16 & 26.09 & 1.71 & 0.70 & 1.42 \\
\hline 79 & Sapindus mukorossi & Ex & 1 & 0 & 0 & 1 & 4.35 & 0.29 & 0.04 & 0.09 \\
\hline 80 & Sesbania grandiflora & Ex & 0 & 0 & 2 & 2 & 4.35 & 0.29 & 0.09 & 0.18 \\
\hline 81 & Spathedea campanulata & Ex & 16 & 2 & 0 & 18 & 26.09 & 1.71 & 0.78 & 1.60 \\
\hline 82 & Swietenia macrophylla & Ex & 24 & 0 & 3 & 27 & 26.09 & 1.71 & 1.17 & 2.39 \\
\hline
\end{tabular}


Table 5. Cont.

\begin{tabular}{|c|c|c|c|c|c|c|c|c|c|c|}
\hline $\begin{array}{l}\text { Sl. } \\
\text { No. }\end{array}$ & List of Species & Origin & Urban & Transition & Rural & Total & Frequency & $\mathbf{R F}$ & Density & RD \\
\hline 83 & Swietenia mahagoni & Ex & 16 & 0 & 0 & 16 & 13.04 & 0.86 & 0.70 & 1.42 \\
\hline 84 & Syzygium cumini & $\mathrm{Na}$ & 10 & 1 & 3 & 14 & 26.09 & 1.71 & 0.61 & 1.24 \\
\hline 85 & Syzygium jambos & Ex & 0 & 1 & 0 & 1 & 4.35 & 0.29 & 0.04 & 0.09 \\
\hline 86 & Tabebuia aurea & Ex & 2 & 0 & 0 & 2 & 4.35 & 0.29 & 0.09 & 0.18 \\
\hline 87 & Tabebuia rosea & Ex & 16 & 0 & 1 & 17 & 17.39 & 1.14 & 0.74 & 1.51 \\
\hline 88 & Tamarindus indica & Ex & 0 & 0 & 2 & 2 & 8.70 & 0.57 & 0.09 & 0.18 \\
\hline 89 & Tecoma stans & Ex & 22 & 1 & 1 & 24 & 26.09 & 1.71 & 1.04 & 2.13 \\
\hline 90 & Tectona grandis & $\mathrm{Na}$ & 2 & 10 & 34 & 46 & 56.52 & 3.71 & 2.00 & 4.08 \\
\hline 91 & Terminalia catappa & $\mathrm{Na}$ & 4 & 1 & 1 & 6 & 26.09 & 1.71 & 0.26 & 0.53 \\
\hline 92 & Thespesia populnea & $\mathrm{Na}$ & 11 & 0 & 1 & 12 & 17.39 & 1.14 & 0.52 & 1.06 \\
\hline 93 & Thuja biota & Ex & 0 & 1 & 0 & 1 & 4.35 & 0.29 & 0.04 & 0.09 \\
\hline \multirow[t]{2}{*}{94} & Unknown & Un & 1 & 0 & 0 & 1 & 4.35 & 0.29 & 0.04 & 0.09 \\
\hline & Total & & 496 & 180 & 452 & 1128 & 1521.74 & 100 & 49.04 & 100 \\
\hline
\end{tabular}

Origin: Native (Na), Exotic (Ex) and Unknown (Un). Except Leucaena leucocephala, Muntingia calabura and Prosopis juliflora all the exotics are naturalized.

Table 6. Importance Value Index (IVI) along northern transect.

\begin{tabular}{|c|c|c|c|c|}
\hline Species & Origin & Urban & Transition & Rural \\
\hline Areca catechu & $\mathrm{Na}$ & & 7.86 & \\
\hline Artocarpus heterophyllus & $\mathrm{Na}$ & 8.07 & 7.86 & 10.99 \\
\hline Azadirachta indica & $\mathrm{Na}$ & 6.18 & 16.26 & 11.33 \\
\hline Cocos nucifera & Ex & 39.31 & 58.18 & 59.02 \\
\hline Dilonix regia & Ex & 6.59 & & \\
\hline Duranta plumeri & Ex & & 6.74 & \\
\hline Eucalyptus hybrid & Ex & & 11.75 & 14.75 \\
\hline Ficus benghalensis & $\mathrm{Na}$ & & & 7.62 \\
\hline Ficus glomerata & $\mathrm{Na}$ & & 6.74 & \\
\hline Ficus religiosa & $\mathrm{Na}$ & & 10.09 & 51.89 \\
\hline Ficus tinctoria & $\mathrm{Na}$ & & & 8.02 \\
\hline Grevillea robusta & Ex & 10.09 & 14.56 & 15.50 \\
\hline Mangifera indica & $\mathrm{Na}$ & 13.99 & 11.79 & 7.54 \\
\hline Michelia champaca & $\mathrm{Na}$ & 6.59 & & \\
\hline Moringa oleifera & $\mathrm{Na}$ & & 7.86 & \\
\hline Morus alba & Ex & & 12.87 & \\
\hline Muntingia calabura & Ex & & 6.74 & \\
\hline Murraya koenigii & $\mathrm{Na}$ & & 7.86 & 7.55 \\
\hline Peltophorum pterocarpum & $\mathrm{Na}$ & 11.17 & & \\
\hline Polyalthia longifolia & Ex & 20.87 & & \\
\hline Pongamia pinnata & $\mathrm{Na}$ & 16.28 & 21.27 & 8.12 \\
\hline Psidium guajava & Ex & & 10.67 & \\
\hline Samanea saman & Ex & 6.46 & & \\
\hline Spathedea campanulata & Ex & 9.82 & & \\
\hline Swietenia macrophylla & Ex & 13.05 & & \\
\hline Swietenia mahagoni & Ex & 8.48 & & \\
\hline Syzygium cumini & $\mathrm{Na}$ & 6.72 & & \\
\hline Tabebuia rosea & Ex & 8.48 & & \\
\hline Tecoma stans & Ex & 11.57 & & \\
\hline Tectona grandis & $\mathrm{Na}$ & & 16.26 & 16.79 \\
\hline Thespesia populnea & $\mathrm{Na}$ & 6.46 & & \\
\hline
\end{tabular}

Origin: Native (Na), Exotic (Ex) and Unknown (Un). Except Muntingia calabura all the exotics are naturalized.

\subsection{Tree Variables}

\subsubsection{Height}

The height of the trees varied from $2.8 \mathrm{~m}$ to $22.5 \mathrm{~m}, 3 \mathrm{~m}$ to $20.3 \mathrm{~m}$ and $2.5 \mathrm{~m}$ to $24.3 \mathrm{~m}$ in rural, transition and urban plots, respectively. The highest number of trees, with 199 trees, were found in the range from $11 \mathrm{~m}$ to $15 \mathrm{~m}$. A total of 179 and 74 trees from the rural and transition plots, respectively, fell in the range from $6 \mathrm{~m}$ to $10 \mathrm{~m}$, with an average height of 
$8.01 \mathrm{~m}$ and $7.57 \mathrm{~m}$. Similarly, 199 trees from the urban plots were found in the range from $11 \mathrm{~m}$ to $15 \mathrm{~m}$, with an average height of $13.12 \mathrm{~m}$. The fewest number of trees were found in the range from $21 \mathrm{~m}$ to $25 \mathrm{~m}$, with an average height of $21.9 \mathrm{~m}$ and $21.77 \mathrm{~m}$ from rural and urban plots, respectively.

\subsubsection{Crown Base Height and Crown Height}

The average crown base height of the trees from rural, transition and urban plots was $4.86 \mathrm{~m}, 4.77 \mathrm{~m}$ and $5.42 \mathrm{~m}$, respectively. The maximum number of trees from all of the three domain (rural, transition, urban) plots was found in the range from $0 \mathrm{~m}$ to $4 \mathrm{~m}$, which shows that the stem part of the tree was visible only a few meters above the ground.

Out of 496 urban plot trees, 180 transition plot trees and 452 rural plot trees, 319, 129, and 289 trees ranged between $3.1 \mathrm{~m}$ and $9 \mathrm{~m}$, with an average crown height of $6.12 \mathrm{~m}$, $5.62 \mathrm{~m}$ and $6.04 \mathrm{~m}$, respectively. The crown heights above $18.1 \mathrm{~m}$ were found only in rural plots with a height of $19 \mathrm{~m}$.

\subsubsection{Crown Shape, Density and Symmetry}

The maximum number of trees was classified under paraboloid and vertical ellipsoid along the gradient.

Along the gradient of the northern transact, irrespective of their domain, most of the tree crowns were non-symmetric. Most of the trees were classified as medium (40-80\%) and sparse (0-40\%). Few trees had dense crowns (80-100\%).

\subsubsection{Tree Access}

The tree access was categorized to understand the status of the trees as street trees or private/garden trees. The ratio of trees almost remains equal between the categories towards the urban domain. However, the private/farm trees were more towards the rural and transition domain.

The trees were classified as solitary and trees in a patch, as on the field, few of the tree crowns were so compact to delineate on the satellite image. Irrespective of the domain, the maximum number of trees was found to be solitary.

\subsubsection{Tree Permat and Tree Condition, Basal Area and Volume}

Tree permat describes the pavement around the tree. In the rural plots, the maximum number of trees was planted in bare soil. Gradually, the trees were found with a nonpermeable pavement towards the transition and urban plots.

On visual analysis, maximum trees were classified as healthy trees with whole or partial crown visibility along the gradient. Dead and declining trees were found towards the transition and urban domain, probably due to the stress on trees due to pollution.

The basal areas of the northern transact increased from $96.39 \mathrm{~m}^{2}$ to $102.76 \mathrm{~m}^{2}$, with a gain of $6.37 \mathrm{~m}^{2}$ from 2017 to 2019 . Similarly, the wood volume increased from $1819.57 \mathrm{~m}^{3}$ to $1926.23 \mathrm{~m}^{3}$, with a gain of $106.66 \mathrm{~m}^{3}$ along the transect. A total of 127 trees were cut, which reduced the tree species to 83, with 1001 individual trees along the gradient. A total of 71, 26 and 30 trees were cut along the rural, transition and urban plots, respectively (Table 7). The loss in the basal area with a reduction in trees over consecutive years was higher in urban plots with $3.66 \mathrm{~m}^{2}$, followed by rural plots with $2.11 \mathrm{~m}^{2}$. Though the loss in trees was higher in rural plots compared to transition and urban plots, a greater change in basal area and volume was observed in urban plots with the reduction in higher girth trees. This indicates that the bigger trees are found more in urban compared to transition and rural plots. 
Table 7. Species change pattern along the northern transect of urban-rural gradient in Bengaluru.

\begin{tabular}{|c|c|c|c|c|c|}
\hline Domain & Parameters & 2017 & 2018 & 2019 & * Change \\
\hline \multirow[t]{4}{*}{ Urban } & Number of Species & 64 & 58 & 55 & -9 \\
\hline & Number of trees & 496 & 474 & 466 & -30 \\
\hline & Basal area $\left(\mathrm{m}^{2}\right)$ & 43.01 & 44.56 & 46.67 & 3.66 \\
\hline & Wood volume $\left(\mathrm{m}^{3}\right)$ & 803.47 & 827.35 & 862.17 & 58.7 \\
\hline \multirow[t]{4}{*}{ Transition } & Number of Species & 37 & 32 & 31 & -6 \\
\hline & Number of trees & 180 & 161 & 154 & -26 \\
\hline & Basal area $\left(\mathrm{m}^{2}\right)$ & 12.8 & 12.75 & 13.4 & 0.6 \\
\hline & Wood volume $\left(\mathrm{m}^{3}\right)$ & 233.56 & 233.87 & 245.23 & 11.67 \\
\hline \multirow[t]{4}{*}{ Rural } & Number of Species & 56 & 53 & 51 & -5 \\
\hline & Number of trees & 452 & 421 & 381 & -71 \\
\hline & Basal area $\left(\mathrm{m}^{2}\right)$ & 40.58 & 42.13 & 42.69 & 2.11 \\
\hline & Wood volume $\left(\mathrm{m}^{3}\right)$ & 782.54 & 810.52 & 818.83 & 36.29 \\
\hline \multirow[t]{4}{*}{ Total } & Number of Species & 93 & 87 & 83 & -10 \\
\hline & Number of trees & 1128 & 1056 & 1001 & -127 \\
\hline & Basal area $\left(\mathrm{m}^{2}\right)$ & 96.39 & 99.44 & 102.76 & 6.37 \\
\hline & Wood volume $\left(\mathrm{m}^{3}\right)$ & 1819.57 & 1871.74 & 1926.23 & 106.66 \\
\hline
\end{tabular}

* change in northern transect during 2017 to 2019.

\section{Discussion}

Our present study focused on tree species change patterns along the urban-rural gradient of the rapidly growing megacity Bengaluru (India) from the stratum "settlement areas", where WorldView-3 imagery supported the selection. The study addressed the varied species composition, distribution, density, frequency and diversity (Shannon index, Simpson index, Pielou's evenness and Margalef's richness) of the trees in the urban, the transition and the surrounding rural domain of Northern Bengaluru. In addition, basal area and wood volume were calculated to understand the biomass potential of the trees in each plot and a total of eleven tree variables were observed for each tree mapped along the gradient.

The study showed that the tree diversity indices indicated a decline towards transition and rural plots, representing that the tree species were more diverse with species composition and distribution in urban plots. However, a sharp decline was observed towards transition and rural plots for the Shannon-Wiener and Margalef indexes. A similar trend was observed for the percentage of exotic species, which explained the rapidly expanding urbanization in combination with land-use changes along the urban-rural gradient. Importance value index (IVI) values also indicated the significance of ornamental tree species planted in an urban domain for beautification of the landscape, whereas religious/multipurpose trees species were found towards rural and transition domains. Comparatively, urban plots were found with taller and larger trees than transition and rural trees. Few trees in transition and rural areas had widespread canopy because they are solitary in nature. On visual analysis, trees in urban plots were found to be in stress with some dead/declining trees compared to rural and transition plots.

Prior studies on tree diversity are limited to urban vegetation in tropical countries [26,27], particularly in India [28]. Recently, few studies have noted high species richness in cities that include rare species that are absent in the surrounding areas [29]. According to [30], urbanization has led to species extinction, which often leads to a negative impact on existing plant diversity. This, in turn, results in replacing native species with more widely distributed non-native species and thus promotes biotic homogenization [31]. According to [10], the decline in the number of species per $\mathrm{km}^{2}$ was observed, with only $25 \%$ of native plant species currently present in the urban areas. Further, the construction of cities and expansion of urban areas also promote the replacement of native species by non-native species [30]. McKinney [30] also showed the increasing intensity of urban activity has resulted in an increase in abundance and species richness of non-native species over native 
species. McKinney [32] stated that non-native plant species are often planted in urban and transition areas. According to Nagendra [33], the greater loss in green areas was observed in transition and rural areas compared to urban areas due to the availability of large open spaces leading to unplanned and unidirectional urbanization. In the present study, Cocos nucifera was the most frequently occurring species in all three domains in Bengaluru due to its religious significance (https: / / www.mangalparinay.com/blog/indiantraditions/importance-and-significance-of-coconut-in-indian-culture) (assessed on $16 \mathrm{De}-$ cember 2021), favorable site conditions and, most importantly, its high tradition uses in households. The urban domain had the highest frequency of ornamental and shade tree species, whereas rural domain had timber, multi-purpose and religious tree species. In the transition area, the most frequent species included a combination of timber, ornamental and shade trees species indicating the characteristics of both the urban and the rural domain.

According to McDonell [34], the species richness along the rural-urban gradient depended on the species concerned, but trees often increase towards the city center. Studies by Mutlu [35], Roy and Mukherjee [36], Vakhlamova [37], Nagendra and Gopal [6,12] also showed similar results of the dominance of exotic species over native species in the urban regions. Our results were found in contrast with the study by McKinney [32], which stated that compared to rural areas, the urban region has lower species diversity. However, he specified over increased patchiness and domination by non-native species in urban areas, which is similar to our results. Shannon's index in the present study was in accordance with Nagendra and Gopal [6]. The tree species observed in our study were also similar to the results presented by Nagendra and Gopal [6,12] and Ramachandra [38].

\section{Conclusions}

The study focused on the changing tree species pattern along the urban-rural gradient in Bengaluru, India. The study is important for the effective management and planning of vegetation within the city. It provides planners and the general public with tree species information, which helps in the selection of adaptive species while designing for the urban plantation. Further, the urban corridors can be planned for the city for conserving the urban biodiversity. The corridor strongly helps in increasing the species richness and habitat quality. However, an increase in the number of samplings along the rural-urban gradient can assist in knowing the pattern of species diversity more accurately. In addition, our sampling approach turned out to be straight forward, and the sample plots laid out can serve as permanent preservation plots for the students, researchers and city planners for regular monitoring and data collection of trees and studying dynamics of tree cover in coming years.

Author Contributions: B.N.D.-Executed the work in the field and drafted manuscript; C.U.N.Helped in collection and compilation of data and drafting of the manuscript; V.P.T.-Helped in the execution of the work and lesioning with German counterparts and edited the manuscript; N.N. and C.K., As German counterparts helped in procurement and finalization of GIS maps, they vetted the manuscript and provided valuable suggestions for improving the draft. All authors have read and agreed to the published version of the manuscript.

Funding: This work was supported by the Department of Biotechnology (DBT), New Delhi (India).

Institutional Review Board Statement: Not applicable.

Informed Consent Statement: Not applicable.

Data Availability Statement: All data is available on request.

Acknowledgments: We thank the German Research Foundation (DFG) for providing the funds to purchase Word View 3 images in the framework of project FOR2432. The authors are also grateful to the Director, Institute of Wood Science and Technology (IWST) for providing all the necessary facilities for the study. The study was funded by the Department of Biotechnology (DBT), GOI, under Indo-German collaboration, which is gratefully acknowledged.

Conflicts of Interest: The authors declare that they have no competing interest. 


\section{References}

1. UNDESA. World Urbanization Prospects: The 2018 Revision, New York 2019; 126p. Available online: https://population.un.org/ wup/Publications / Files/WUP2018-Report.pdf (accessed on 16 December 2021).

2. Sudhira, H.S.; Ramachandra, T.V.; Subrahmanya, M.H.B. City profile Bangalore. Cities 2007, 24, 379-390. [CrossRef]

3. Narayana, M.R. Globalization and Urban Economic Growth: Evidence for Bangalore, India. Int. J. Urban Reg. Res. 2011, 35, 1284-1301. [CrossRef]

4. $\quad$ Ramachandra, T.V.; Aithal, B.H.; Durgappa, D.S. Insights to urban dynamics through landscape spatial pattern analysis. Int. J. Appl. Earth Obs. Geoinf. 2012, 18, 329-343.

5. Bangalore, India Metro Area Population 1950-2021. Available online: https://www.macrotrends.net/cities/21176/bangalore/ population (accessed on 16 December 2021).

6. Nagendra, H.; Gopal, D. Street trees in Bangalore-Density, diversity, composition and distribution. Urban For. Urban Green. 2010, 9, 129-137. [CrossRef]

7. Gopal, D.; Nagendra, H. Vegetation in Bangalore's slums: Boosting livelihoods, well-being and social capital. Sustainability 2014, 6, 2459-2573. [CrossRef]

8. Nagendra, H.; Lucas, R.; Honrado, J.P.; Jongman, R.H.G.; Tarantino, C.; Adamo, M.; Mairota, P. Remote sensing for conservation monitoring: Assessing protected areas, habitat extent, habitat condition, species diversity and threats. Ecol. Indic. 2013, 33, 45-59. [CrossRef]

9. Paul, S.; Nagendra, H. Vegetation change and fragmentation in the mega city of Delhi: Mapping 25 years of change. Appl. Geogr. 2015, 58, 153-166. [CrossRef]

10. Aronson, M.F.; La Sorte, F.A.; Nilon, C.H.; Katti, M.; Goddard, M.A.; Lepczyk, C.A.; Warren, P.S.; Williams, N.S.; Cilliers, S.; Clarkson, B.; et al. A global analysis of the impacts of urbanization on bird and plant diversity reveals key anthropogenic drivers. Proc. R. Soc. B Biol. Sci. 2014, 281, 20133330. [CrossRef]

11. Aronson, M.F.J.; Handel, S.N.; La Puma, I.P.; Clemants, S.E. Urbanization promotes non-native woody species and diverse plant assemblages in the New York metropolitan region. Urban Ecosyst. 2015, 18, 31-45. [CrossRef]

12. Nagendra, H.; Gopal, D. Tree diversity, distribution, history and change in urban parks: Studies in Bangalore, India. Urban Ecosyst. 2011, 14, 211-223. [CrossRef]

13. Chaudhry, P.; Bagra, K.; Singh, B. Urban Greenery Status of Some Indian Cities: A Short Communication. Int. J. Environ. Sci. Dev. 2011, 2, 98-101. [CrossRef]

14. Gowda, K.; Sridhara, M.V.; Rajan, S. Planning and management of parks and green areas: The case of Bangalore metropolitan area. Manag. Environ. Qual. 2007, 19, 270-282. [CrossRef]

15. Behera, G.; Nageshwara, R.; Dutt, P.P.; Manikiam, B.; Balakrishna, P.; Krishnamurthy, J.; Jagadeesh, K.M.; Ganesha, R.K.; Diwakar, P.G.; Padmavathy, A.S.; et al. Growth of Bangalore City Since 1980 Based on Maps and Satellite Imagery; Technical Report, ISRO-EOS-TR-55-85; Indian Space Research Organization: Bangalore, India, 1985.

16. Sudha, P.; Ravindranath, N.H. A study of Bangalore urban forest. Landsc. Urban Plan. 2000, 47, 47-63. [CrossRef]

17. Maurer, U.; Peschel, T.; Schmitz, S. The flora of selected land-use types in Berlin and Potsdam with regard to nature conservation in cities. Landsc. Urban Plan. 2000, 46, 209-215. [CrossRef]

18. Zerbe, S.; Choi, I.; Kowarik, I. Characteristics and habitats of non-native plant species in the city of Chonju, Southern Korea. Ecol. Res. 2004, 19, 91-98. [CrossRef]

19. Curtis, J.T.; McIntosh, R.P. The interrelations of certain analytic and synthetic phytosociological characters. Ecology 1950, 31 434-455. [CrossRef]

20. Shannon, C.E.; Wiener, W. The Mathematical Theory of Communication; University of Illinois Press: Urbana, IL, USA, 1963; 127p.

21. Simpson, E.H. Measurement of diversity. Nature 1949, 163, 688. [CrossRef]

22. Ludwig, J.A.; Reynolds, J.F. Statistical Ecology; John Wiley and Sons: New York, NY, USA, 1988.

23. Pielou, E.C. Ecological Diversity; John Wiley \& Sons: New York, NY, USA, 1975; viii + 165p.

24. Gentry, A.H. Changes in Plant Community Diversity and Floristic Composition on Environmental and Geographical Gradients. Ann. Mo. Bot. Gard. 1988, 75, 1-34. [CrossRef]

25. Kacholi, D.S. Analysis of Structure and Diversity of the Kilengwe Forest in the Morogoro Region, Tanzania. Int. J. Biodivers. 2014, 2014, 516840. [CrossRef]

26. Jonsson, P. Vegetation as an urban climate control in the subtropical. Int. J. Climatol. 2004, 24, 1307-1322. [CrossRef]

27. Song, X.P.; Richards, D.; Edwards, P.; Tan, P.Y. Benefits of trees in tropical cities. Science 2017, 356, 1241. [CrossRef]

28. Lal, C.B.; Annapurna, C.; Raghubanshi, A.S.; Singh, J.S. Effect of leaf habit and soil type on nutrient resorption and conservation in woody species of a dry tropical environment. Can. J. Bot. 2001, 79, 1066-1075.

29. Godefroid, S.; Koedam, N. Urban plant species patterns are highly driven by density and function of built-up areas. Landsc. Ecol. 2007, 22, 1227-1239. [CrossRef]

30. McKinney, M.L. Urbanization as a major cause of biotic homogenization. Biol. Conserv. 2006, 127, 247-260. [CrossRef]

31. Olden, J.D.; Poff, N.L. Toward a mechanistic understanding and prediction of biotic homogenization. Am. Nat. 2003, 162, 442-460 [CrossRef] [PubMed] 
32. McKinney, M.L. Urbanization, biodiversity, and conservation: The impacts of urbanization on native species are poorly studied, but educating a highly urbanized human population about these impacts can greatly improve species conservation in all ecosystems. Bioscience 2002, 52, 883-890. [CrossRef]

33. Nagendra, H.; Nagendran, S.; Paul, S.; Pareeth, S. Graying, greening and fragmentation in the rapidly expanding Indian city of Bangalore. Landsc. Urban Plan. 2012, 105, 400-406. [CrossRef]

34. McDonnell, M.J.; Hahs, A.K. The use of gradient analysis studies in advancing our understanding of the ecology of urbanizing landscapes: Current status and future directions. Landsc. Ecol. 2008, 23, 1143-1155. [CrossRef]

35. Mutlu, S.S.; Selim, C.; Gülçin, Ü.N. Plant Biodiversity of Urban Roadside Trees in Antalya, Turkey. J. For. Fac. $2017,17,80-87$. [CrossRef]

36. Roy, D.; Mukherjee, S.K. Diversity of trees in Kalyani township in West Bengal. J. Econ. Taxon. Bot. 2011, 35, 687-695.

37. Vakhlamova, T.; Rusterholz, H.P.; Kamkin, V.A.; Baur, B. Recreational use of urban and suburban forests affects plant diversity in a Western Siberian city. Urban For. Urban Green. 2016, 17, 92-103. [CrossRef]

38. Ramachandra, T.V.; Aithal, B.H.; Kulkarni, G.; Vinay, S. Green spaces in Bengaluru: Quantification through geospatial techniques. Indian For. 2017, 143, 307-320. 